\title{
Diffusion constant for the repton model of gel electrophoresis
}

\author{
M. E. J. Newman \\ Santa Fe Institute, 1399 Hyde Park Road, Santa Fe, New Mexico 87501 \\ G. T. Barkema \\ HLRZ, Forschungszentrum Jülich, 52425 Jülich, Germany
}

(Received 10 March 1997)

\begin{abstract}
The repton model is a simple lattice model of the "reptation" motion by which DNA diffuses through a gel during electrophoresis. In this paper we show that the model can be mapped onto a system consisting of two types of particles with hard-sphere interactions diffusing on a one-dimensional lattice. Using this mapping we formulate an efficient Monte Carlo algorithm for the model which allows us to simulate systems more than twice the size of those studied before. Our results confirm scaling hypotheses which have previously been put forward for the model. We also show how the particle version of the model can be used to construct a transfer matrix which allows us to solve exactly for the diffusion constant of small repton systems. We give results for systems of up to 20 reptons. [S1063-651X(97)13909-5]
\end{abstract}

PACS number(s): 87.10.+e, 82.45.+z

\section{INTRODUCTION}

Gel electrophoresis is an experimental tool of great importance in the ever-growing industries of genetics and polymer research. It allows an experimenter to separate a mixture of polymer strands by length or, in combination with sample strands of known lengths, to measure the lengths of strands in the mixture. However, despite the development of sophisticated techniques for exploiting electrophoresis in the laboratory, the physics behind the method is still imperfectly understood. A number of efforts have been made to model the diffusion of polymers in gels, with varying degrees of success. In this paper we examine one of the simplest but also most successful of these, the repton model, which is primarily of use as a model of DNA electrophoresis in agarose gels. (It does not model the behavior of most other polymers very well, nor the behavior of DNA in polyacrylomide gels.)

Although the subject of many functional refinements and improvements over the years, the basic DNA agarose-gel experiment is in essence a very simple one. A gel is formed by adding agarose powder to a warm buffer solution, pouring the mixture into the gel box, and allowing it to cool, whereupon the agarose strands cross-link to form a threedimensional web with a typical pore size of about $1000 \AA$. The DNA is placed in solution with more of the same buffer, from which it gains an electric charge, and is then injected into the gel. An electric field, typically on the order of a few volts per centimeter, is applied horizontally across the box, and the charged DNA migrates under its influence through the gel. It is found that the rate of migration depends principally on three factors: the pore size of the gel (which in turn depends on the concentration of the original agarose solution), the magnitude of the applied electric field, and, crucially, the length of the DNA strands. Longer DNA strands are impeded more by the agarose in the gel and travel slower, so that after the experiment has been running for some time (typically a few hours), the initially homogeneous DNA mixture will have separated out along a "lane" in the gel box, according to the lengths of its constituent parts. The distance traveled by any particular component of the mixture is a measure of its length, and the final gel can be dissected into pieces to refine the DNA by length.

The basic mechanism responsible for the length dependence of the migration rate was first described by de Gennes [1]. The important point is that the persistence length of DNA under the conditions of the experiment is between about 400 and $800 \AA$, which is of the same order of magnitude as the pore size of the gel. This means that the agarose strands in the gel effectively prevent movement of a strand of DNA transversely to its length, so that it migrates primarily by a longitudinal slithering motion, for which de Gennes coined the term "reptation." The repton model is one of the simplest models of the reptation process. It was invented in 1987 by Rubinstein [2] as a model of the dynamics of entangled polymers, and co-opted as a model of DNA reptation by Duke [3]. Briefly the model is as follows. (A more thorough exposition can be found, for example, in Ref. [4].)

The repton model simulates the movement of a single strand of DNA through the gel. The strand is represented as a number $N$ of points, joined together by lines-see Fig. 1(a). The points are confined to the squares of a twodimensional rectangular lattice which is represented by the grid of lines in the figure. The points in the strand are known as "reptons," and successive reptons in the strand may lie either in the same square, or in adjacent ones, but may not be further apart than this. This ensures that the DNA has some elasticity but is not infinitely stretchy. The lattice squares represent the pores in the agarose gel, so that the lattice parameter $a$ is also the pore size. The distance between successive reptons in the chain represents the persistence length of the DNA (a few hundred base pairs, or about 400 to 800 $\AA$, as mentioned above), and since this is limited to at most one lattice spacing, it is implicit in the model that the persistence length and the pore size are approximately equal. (It is for this reason that the repton model makes a poor approximation to the behavior of DNA in other types of gel, or of other polymers in agarose.)

The use of a two-dimensional lattice in the model appears 
at first to be problematic. Clearly the real DNA moves in a three-dimensional space. However, as we will see in Sec. II, the behavior of the model is actually independent of the dimensionality of the lattice, so a two-dimensional one is as good as any other.

In order to simulate the reptation motion of the DNA, the following dynamics is imposed on the repton chain.

(i) A repton in the interior of the chain may move to one of the adjacent squares on the lattice, provided that one of its immediate neighbors in the chain is already in the square to which it is moving, and the other is in the square which it leaves.

(ii) The two reptons at the ends of the chain may move in any direction to an adjacent square, provided that such a move does not take them more than one lattice spacing away from their neighboring repton on the chain. These two rules ensure that the chain always diffuses along its own length, and that neighboring reptons on the chain are never more than one lattice spacing apart. The time-scale for the model is set by stipulating that each repton, driven by random thermal fluctuations, should attempt to move in each of the four possible directions once on average per unit time. Each attempted move is accepted and carried out if it is allowed by the rules above, otherwise it is rejected and the chain stays as it was.

Notice that all allowed moves proceed at the same rate; there are no energies in the repton model and no Boltzmann weights. The model is entirely entropy-driven. If we wish to model the migration of the DNA in the applied electric field we need to introduce a bias in the selection probabilities for moves in one particular direction, usually the $x$ axis. However, if, as the experimentalists are, we are primarily interested in the rate $v$ of migration in a given electric field $E$, it is not necessary to introduce such a bias. The value of $v$ in the low-field limit can be calculated instead from the zerofield diffusion constant $D$ using the Nernst-Einstein relation:

$$
D=\lim _{E \rightarrow 0} \frac{v}{N E} .
$$

This is the approach we will be taking in this paper, and our calculations will concentrate on the evaluation of the diffusion constant, in the knowledge that the value of $v$ can always be calculated from it in a simple fashion. [Equation (1) should be valid as long as the product $N E$ of the chain length and the electric field strength is below a certain value [5]. For DNA fragments of lengths for which agarose is the gel medium of choice, this limits the electric field to about 10 $\mathrm{V} / \mathrm{cm}$, and experiments have confirmed this figure [6]. Typical laboratory procedure employs fields comfortably within this range, implying that Eq. (1) should be an acceptable approximation.]

The repton model ignores many important features of the dynamics of the real DNA, such as excluded volume effects due to the finite space occupied by the DNA, self-repulsion effects due to its charge, the effects of counterions, mechanical properties of the DNA, and inhomogeneities in the gel. Surprisingly, however, it gives results in fairly good agreement with experiment [5], leading us to believe that it may well capture many of the essential features of the dynamics of DNA in agarose.
Some of the earliest analytic work on the repton model was performed by van Leeuwen and Kooiman [7], who showed that under the assumption of periodic boundary conditions the limiting value of $D$ as $N \rightarrow \infty$ is $1 /\left(3 N^{2}\right)$. Later, Prähofer [8] demonstrated rigorously that this limiting behavior holds also for the repton model without periodic boundary conditions. In practice, however, the large finitesize effects present in the model mean that the measured value of $D$ is far from this limit. In our calculations we usually quote figures for $D N^{2}$, which should have a limiting value of $\frac{1}{3}$.

In 1991, Widom et al. [9] took an important step forward by showing that it was possible to solve the repton model exactly for finite values of the chain length $N$ using a combination of a transfer matrix method with a perturbation theoretic analysis. The matrix used is essentially the stochastic or Markov matrix for the dynamics of the model, which has one row and one column for each possible state of the chain. As discussed in Sec. IV, this matrix, in its most compact form, has rank $3^{N-1}$. Unfortunately this makes the required diagonalization operation prohibitively costly for all but the shortest repton chains. Widom et al. carried out the calculation for values of $N$ up to 5, and these results were later extended by Szleifer and Bisseling to $N=12$ using a special-purpose computer [10].

In this paper we propose a mapping of the repton model onto a one-dimensional particle model with two types of particles possessing hard-sphere repulsion. Using this mapping we show that the repton model can be solved exactly by finding only the one eigenvector corresponding to the largest eigenvalue of a much smaller matrix, one with rank $(N+1) 2^{N-2}$. Given the relatively conservative size of this matrix and the fact that finding one eigenvector is a lot simpler than diagonalizing the whole matrix, we have been able using this method to extend the exact solution of the model to chains of up to twenty reptons (corresponding to DNA strands of about $4 \mathrm{~Kb}$ ), using only conventional computing resources.

Of the numerical studies which have been performed on the model, probably the most comprehensive to date are those of Barkema, Marko, and Widom [11], who used a multispin coded algorithm running on a supercomputer to simulate the model for values of $N$ up to 100 . Our projection of the model onto a one-dimensional particle system has also led us to a more efficient Monte Carlo algorithm for the model's simulation, which has allowed us to extend these simulation results to $N=250$, again using only conventional computing resources.

The outline of this paper is as follows. In Sec. II, we describe the mapping introduced by Duke [3] of the repton model onto a one-dimensional chain model, and then introduce our further mapping onto a model containing two types of particles diffusing on a one-dimensional lattice. In Sec. III we describe our simulations of this version of the model. In Sec. IV we make use of the mapping to define our reduced transfer matrix and from that matrix extract exact results for the diffusion constant of the model for values of $N$ up to 20 . We also speculate on the connection between the particle version of the repton model and the so-called "asymmetric exclusion models." Some simple versions of these models have been solved analytically for all $N[12,13]$, which leads 


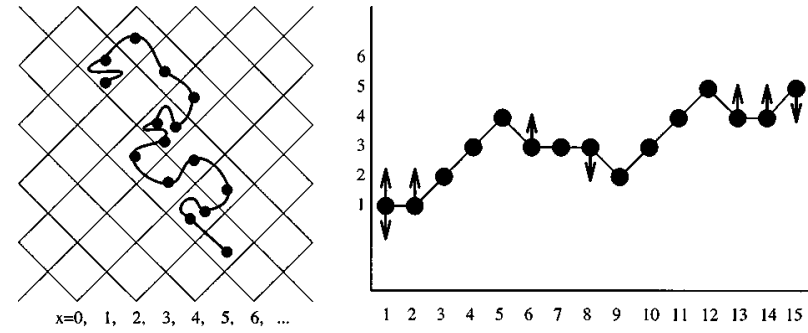

FIG. 1. (a) The repton model of gel electrophoresis. The points-reptons-are connected together in a chain, and consecutive points in the chain must occupy adjacent squares on the lattice, or the same square. (b) The projected repton model described in Sec. II. The arrows indicate the possible moves.

us to hope that a similar solution of the repton model may be possible. In Sec. V we give our conclusions.

\section{THE PROJECTED REPTON MODEL}

The fundamental quantity which we would like to calculate using the repton model is the rate of migration of DNA, as a function of its length, under the influence of an electric field $E$ applied, for example, horizontally in Fig. 1(a). As we pointed out in the preceding section, this can be calculated using the Nernst-Einstein relation, Eq. (1), from a knowledge of the diffusion constant for the diffusion of the repton chain in zero field. Since we are only interested in the movement of the chain along one axis, in this case the $x$ axis, it is only necessary to consider the $x$ component of each repton's position. In Fig. 1(b) we have plotted this $x$ component for the state of the chain depicted in Fig. 1(a) as a function of position along the chain from one end to the other, and this plot contains all the information we need about that state. This projected form of the repton model was first introduced by Duke [3], and it is this form which was used by Widom et al. to construct their transfer matrix, and also by Barkema et al. to perform simulations of the model's properties.

The restriction that consecutive reptons in the chain can lie only in the same or adjacent squares on the lattice translates to the restriction that the positions $x$ of adjacent reptons in the projected model can differ by at most $a / \sqrt{2}$, although we will find it more convenient to measure $x$ in units of this quantity, so that the values are always integers and adjacent ones may differ by only $+1,0$, or -1 . The dynamics of the projected model in zero electric field also can be derived as a simple projection of the dynamics of the original model:

(i) A repton in the interior of the chain may move up or down by one step provided that one of its two neighbors is already at the level to which it is moving, and the other is at the level which it leaves.

(ii) The two reptons at the ends of the chain may move either up or down, provided this does not take them more than one step away from their neighbors.

As with the two-dimensional version of the model, each possible move of each repton is attempted on average once per unit time, and any attempted move is accepted provided it does not violate any of the rules above. If it does, the move is rejected and the chain remains unchanged.

Notice that this projection of the repton model onto the $x$ axis would work just as well had we started with a lattice

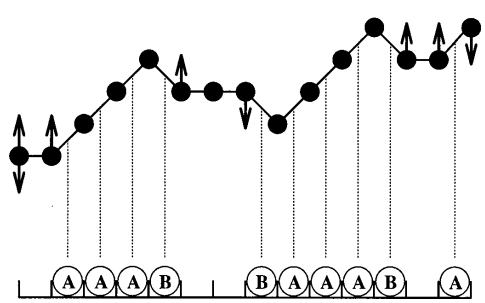

FIG. 2. The mapping of the projected repton model onto a particle model. There are two types of particle, which we label $A$ and $B$. No two particles may coexist on the same lattice site, but otherwise they are free to diffuse about the lattice.

with three or even more dimensions. We conclude that the dynamics of the model is independent of the dimensionality of the original lattice, as we mentioned briefly in the preceding section.

The state of the projected repton model can be specified by giving the $x$ coordinate of each of the $N$ reptons in the chain. Alternatively, as Fig. 1(b) makes clear, we could specify it by describing in turn the $N-1$ links in the chainthe lines between adjacent reptons in the figure-each of which can be in one of three states: sloping upwards to the left, sloping upwards to the right, or level. In order to completely pin the chain down, we would also have to specify the absolute position of one of the reptons - say the leftmost one-but since the model is translationally invariant, all its properties can be calculated without knowledge of this variable. In Fig. 2 we have made use of these new degrees of freedom to create an alternative mapping of the model to a one-dimensional particle model. In this mapping each of the $N-1$ links corresponds to a site on a new lattice, and each site can be in one of three states of occupation, depending on the state of the corresponding link. Sites corresponding to links which slope upwards to the right are occupied by a particle of one type, which we call type $A$, and those corresponding to links which slope upwards to the left are occupied by a different type of particle, type $B$. Sites corresponding to horizontal links are left empty. It is not hard to show that the dynamics of the particles is as follows: (i) Particles in the interior of the chain are conserved; (ii) no two particles may coexist at the same site, regardless of their types; (iii) a particle adjacent to an empty site can move to that site; (iv) a particle at one of the ends of the chain can fall off the chain and vanish; (v) if one of the end sites on the chain is empty, a new particle of either type can appear there. As before, all possible moves are attempted once each on average per unit time. Moves which violate none of the rules above are always accepted. All others are rejected. The particles are in some ways akin to fermions, but their dynamics is completely classical in nature, so we prefer to regard them simply as hard-sphere classical particles.

As far as the diffusion of the repton chain is concerned, we can show that if a particle of type $A$ enters the system at the left-hand end and migrates all the way to the right, and falls off, then the average position of the chain moves one step in the positive $x$ direction. It also moves one step in the positive $x$ direction if a particle of type $B$ moves across the system from right to left. Moves in the opposite directions correspond to motion of the average position in the negative $x$ direction.

By employing this particle mapping of the repton model, 
we have been able to improve considerably on previous calculations of the model, both numerical and exact.

\section{NUMERICAL CALCULATIONS}

If we were to simulate our particle version of the repton model directly, employing the dynamics described above, the resulting calculation would be entirely equivalent to, and no faster than, a direct simulation of the projected repton model. However, we can speed the calculation considerably by observing that, in the zero-field case we are considering here, the dynamics of the two types of particles, $A$ and $B$, is identical. Whether a particular particle is of type $A$ or of type $B$ makes no difference whatsoever to the probability of the system taking a particular path. Thus, it is possible to carry out the entire simulation without assigning any types to any of the particles-we can assign types to them at the end instead. In fact, the best statistics are derived by making in turn every possible assignment of particle types to particles and averaging over all of them. If we denote by $n_{A \rightarrow}$ and $n_{A \leftarrow}$ the number of particles of type $A$ which pass through the system from left to right and from right to left, respectively, during the course of our simulation, and similarly for particles of type $B$, then the mean square distance $\left\langle d^{2}\right\rangle$ traveled by the repton chain in the $x$ direction, where \langle\rangle indicates an average over all possible assignments of particle types, is

$$
\begin{aligned}
\left\langle d^{2}\right\rangle & =\left\langle\left[\left(n_{A \rightarrow}+n_{B \leftarrow}\right)-\left(n_{A \leftarrow}+n_{B \rightarrow}\right)\right]^{2}\right\rangle \\
& =\left\langle\left[\left(n_{A \rightarrow}-n_{B \rightarrow}\right)-\left(n_{A \leftarrow}-n_{B \leftarrow}\right)\right]^{2}\right\rangle \\
& =\left\langle\left(n_{A \rightarrow}-n_{B \rightarrow}\right)^{2}\right\rangle+\left\langle\left(n_{A \leftarrow}-n_{B \leftarrow}\right)^{2}\right\rangle \\
& =\left\langle\left(n_{A \rightarrow}+n_{B \rightarrow}\right)\right\rangle+\left\langle\left(n_{A \leftarrow}+n_{B \leftarrow}\right)\right\rangle \\
& =n_{\rightarrow}+n_{\leftarrow} .
\end{aligned}
$$

The third line here follows from the statistical independence of the numbers of particles of each type, and the fourth line follows from the properties of random walks in one dimension. The variables $n_{\rightarrow}$ and $n_{\leftarrow}$ in the last line are the total numbers of particles passing across the system in each direction during the course of the simulation. The diffusion constant in the $x$ direction is related to $\left\langle d^{2}\right\rangle$ by

$$
D=\frac{\left\langle d^{2}\right\rangle}{2 t},
$$

where $t$ is the length of time (as defined in Sec. I) for which the simulation ran.

In order to calculate $n_{\rightarrow}$ and $n_{\leftarrow}$, we need to count the number of particles falling off the right-hand end of the system which entered from the left, and the number falling off the left which entered from the right. Those which enter and leave at the same end make no contribution. Thus we need to keep a record for each particle in the system of whether it entered at the left- or the right-hand end. We can do this by labeling each with either an " $L$ " or an " $R$," which in effect means that we have two types of particles again.

Using a multispin coded program which simultaneously performs 32 simulations using the algorithm described here, we have calculated the value of the diffusion constant for the repton model for systems of up to $N=250$ reptons. The runs

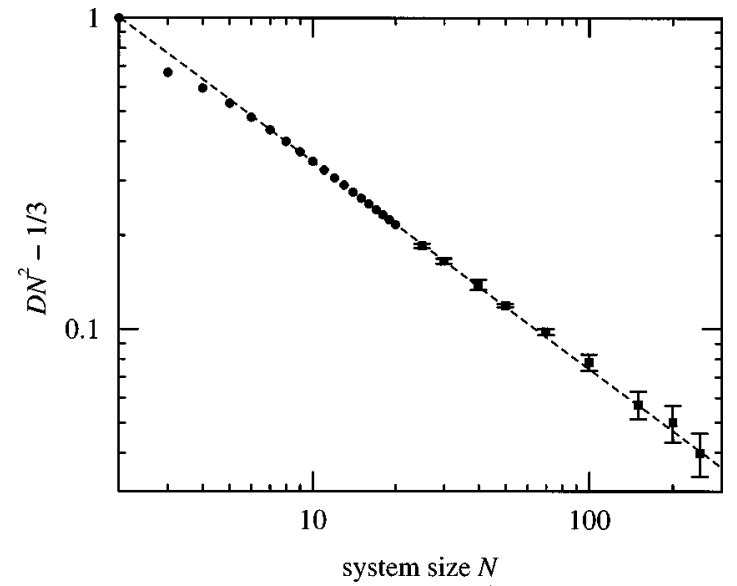

FIG. 3. Results for the diffusion constant $D$ of the repton model for systems of up to $N=250$ reptons. The circles are exact results calculated using the transfer matrix method of Sec. IV. The squares are numerical results obtained by the Monte Carlo method described in Sec. III. We have plotted the data as $D N^{2}-\frac{1}{3}$ against $N$ on logarithmic scales in order to test the scaling form hypothesized in Ref. [11]. The dashed line indicates the slope expected if this scaling form holds.

were of a variety of lengths up to a maximum of around $10^{11}$ Monte Carlo steps for the largest systems. For each run we discarded the first $10 \%$ of the data to allow for equilibration. The results of the simulations are shown in Fig. 3. Barkema, Marko, and Widom [11] have conjectured, on the basis of numerical results for systems of up to $N=100$ reptons, that $D N^{2}-\frac{1}{3}$ scales for large system sizes as $N^{-2 / 3}$. This scaling is indicated as the dashed line in the figure. As the graph shows, our results for values of $N$ above 100 appear to confirm this conjecture.

\section{EXACT CALCULATIONS}

The $L$ - and $R$-type particles introduced in the preceding section are interesting in their own right, since, as we now show, they lead to an exact solution of the model for small values of $N$. Their dynamics is almost the same as that of the $A$ and $B$ particles of the preceding section: they are conserved, can hop either left or right into empty spaces, and particles at the end of the chain can fall off altogether, and all these moves are attempted once on average per unit time. However, if one of the sites at the end of the chain is empty, a new particle appears and fills it on average twice per unit time, rather than once as before. To see this, recall that particles of types $A$ and $B$ both attempted to fill empty end sites once each on average per unit time in our previous version of the model. The total rate of attempted particle entry at either end is therefore two particles per unit time. Other than this, things remain as before: all attempted moves are accepted if they do not violate any of the dynamical rules, otherwise they are rejected.

The total rate then at which particles of type $L$ fall off the right-hand end of the chain is simply one times the density of $L$ particles at the rightmost site, and similarly for particles of type $R$ at the left-hand end. Since the system is, on average, completely left-right symmetric, we can define a density function $\rho(i)$ such that 


$$
\rho_{L}(i)=\rho_{R}(N-i)=\rho(i),
$$

where $\rho_{L}(i)$ and $\rho_{R}(i)$ are the densities of the two types of particles at the $i$ th site (counting, let us say, from the left). Now using Eqs. (2) and (3) we can write the diffusion constant as

$$
D=\frac{n_{\rightarrow}+n_{\leftarrow}}{2 t}=\frac{2 \rho(1) t}{2 t}=\rho(1) .
$$

It would be possible to calculate $\rho(1)$, the density of the minority particles at the first site on the lattice, using a Monte Carlo technique. However, the results for $D$ would be less accurate than those calculated with the methods of the preceding section. On the other hand, Eq. (5) does lend itself to exact calculations. Notice that the number of states of the model with the $L$ and $R$ particles is considerably smaller that the number of states of the one with the $A$ and $B$ particles. The reason is that, since all particles of type $L$ enter from the left, all type $R$ ones from the right, and the two cannot pass one another, all lawful states of the system consist of at most two domains, one on the left containing only $L$ particles and vacancies, and one on the right containing only $R$ particles and vacancies. Thus, once we know the position of the line which separates these two domains, the state of any particular site is completely determined if we know only whether it is occupied or not. There are $N$ possible positions for the line, and hence $N 2^{N-1}$ is an upper bound on the number of states of the system. In fact the actual number of states turns out [14], as we mentioned in Sec. I, to be $(N+1) 2^{N-2}$. This figure is considerably smaller than the $3^{N-1}$ states of the system with the $A$ and $B$ particles, or equivalently of the ordinary projected repton model, which Widom et al. used to construct their transfer matrix. This suggests that it might be possible to construct a new smaller transfer matrix which would allow us to calculate the diffusion constant of the model exactly for larger values of $N$.

The construction of such a matrix turns out to be charmingly straightforward. The matrix possesses one row and one column for each state of the system with the $L$ - and $R$-type particles. Its off-diagonal elements are zero except for a sparse set of elements connecting pairs of states which are accessible to one another via the dynamics of the model. The diagonal elements are fixed to ensure overall particle conservation. The slowest-decaying eigenmode of this matrix represents the equilibrium occupation probabilities of each of the states of the chain, and a simple linear combination of its elements gives the density of particles of either type at any site, the density $\rho(1)$ being a particular case. We have calculated the eigenvector of this mode numerically for systems with sizes from $N=3$ up to $N=20$. Since the matrix is sparse, the quickest method of doing this is simply by repeated multiplication into an initial trial vector. In fact, for each system size studied, we have used two trial vectors, chosen so that the value of $\rho(1)$ calculated from them converges towards the equilibrium value from opposite directions, ensuring that we have rigorous bounds on our values, as well as an absolute measure of convergence. The value of $\rho$ (1) can then be used in Eq. (5) to calculate the diffusion constant. The resultant values for $D N^{2}$ are given in Table I and also shown in Fig. 3. The values for $N$ up to 12 confirm
TABLE I. Values of $D N^{2}$ calculated using the transfer matrix method described in the text. The values are exact to the accuracy quoted.

\begin{tabular}{cccccc}
\hline \hline$N$ & $D N^{2}$ & $N$ & $D N^{2}$ & $N$ & $D N^{2}$ \\
\hline 3 & 1.000000 & 9 & 0.703951 & 15 & 0.596297 \\
4 & 0.926984 & 10 & 0.678924 & 16 & 0.585081 \\
5 & 0.864908 & 11 & 0.657549 & 17 & 0.574996 \\
6 & 0.812065 & 12 & 0.639096 & 18 & 0.565874 \\
7 & 0.769057 & 13 & 0.623006 & 19 & 0.557579 \\
8 & 0.733582 & 14 & 0.608851 & 20 & 0.550001 \\
\hline \hline
\end{tabular}

those obtained by Widom et al. [9] and by Szleifer and Bisseling [10]. Those for $N=13$ up to 20 are new. No errors are given in the table; the figures are exact to the quoted accuracy.

The function $\rho(i)$ is also interesting in its own right. In Fig. 4 we have plotted its value for systems of size $N=20$, 50,100 , and 200 on linear and logarithmic scales, calculated numerically. As $N$ increases, the curves on the linear plot appear to become more and more like a straight line with short curved regions at each end. It seems possible that the asymptotic form of the function is linear, with end effects
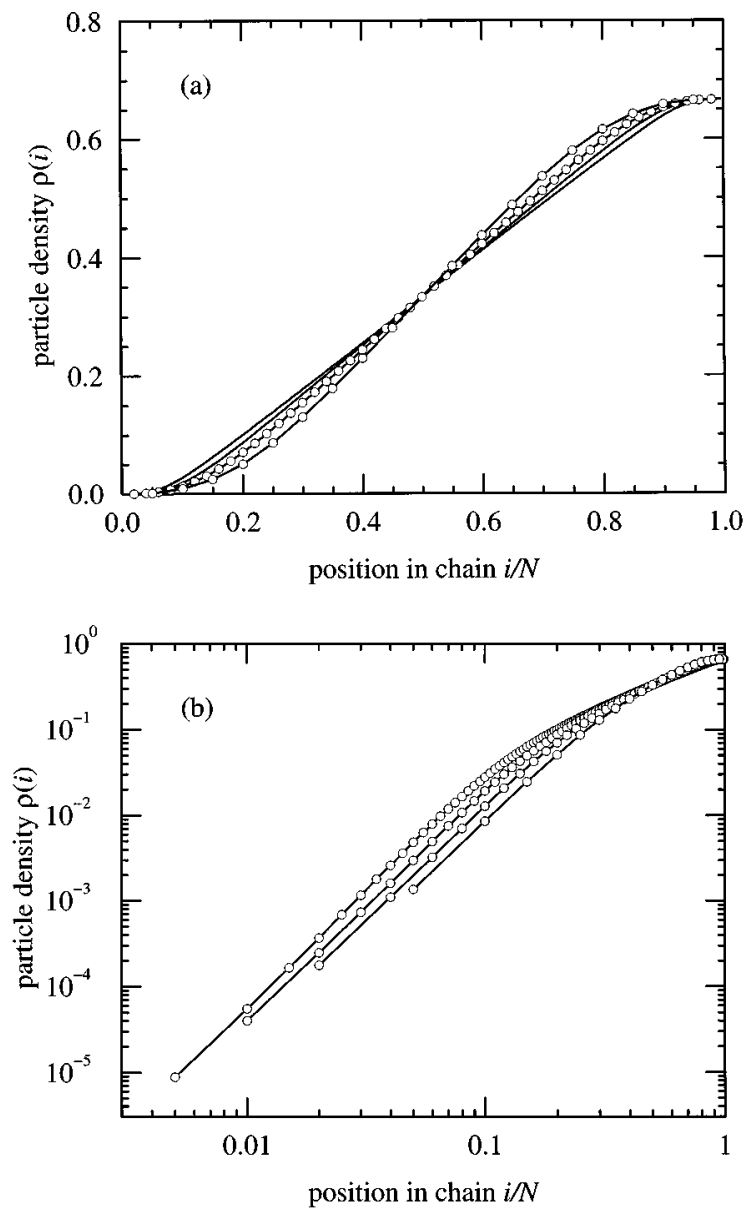

FIG. 4. The function $\rho(i)$ defined in Eq. (4), plotted for systems of size $N=20,50,100$, and 200 on (a) linear scales and (b) logarithmic ones. Close to the end of the repton chain (small $i) \rho(i)$ appears to vary as a power law with $i$. The measured exponent of the power law is $2.76 \pm 0.03$. 
whose region of influence tends to some finite limit. The logarithmic plot sheds further light on the behavior at the ends of the chain. As it shows, $\rho(i)$ appears to become power-law in form for small $i$ as $N$ becomes large. The exponent of the power law is measured to be $2.76 \pm 0.03$.

Consideration of $\rho(i)$ also offers a hint of a possible complete analytic solution of the repton model. The particle version of the model considered here is a particular case of a class of models known as asymmetric exclusion models. In recent work, Derrida et al. [12] and independently Stinchcombe and Schütz [13] have found exact analytic solutions for the density function equivalent to our $\rho(i)$ for a number of models of this class for all values of $N$. The models they solved are simpler in a number of respects than the model studied in this paper, and applying their techniques to our model would not be a trivial task. However, Eq. (5) tells us that, should such a solution prove possible, we would immediately have an expression for the diffusion constant of the repton model for all values of $N$. It is certainly an intriguing prospect.

\section{CONCLUSIONS}

In this paper we have studied the Duke-Rubinstein repton model of DNA reptation in an agarose gel. We have introduced a mapping of the model onto a particle model in which hard-sphere particles hop at random on a finite onedimensional lattice. We have demonstrated that the diffusion constant $D$ of the polymer in the gel is proportional simply to the average rate at which these particles cross from one side of the lattice to the other, and we have made use of this fact to formulate a highly-efficient Monte Carlo algorithm for calculating this diffusion constant. Using this algorithm we have calculated values of $D$ for systems of up to $N=250$ persistence lengths. These results confirm hypotheses put forward earlier concerning the scaling of $D$ with the system size.

We have also employed our particle version of the repton model to construct a transfer matrix whose dominant eigenvector is directly related to the value of the diffusion constant. We have numerically calculated this eigenvector, and hence extracted exact values for $D$ for systems up to $N=20$.

Finally, we have drawn a connection between our particle version of the repton model and the asymmetric exclusion models which have been solved exactly by Derrida et al. and by Stinchcombe and Schütz. We conjecture that it may be possible to use the techniques they employed, along with the theory developed in this paper, to find a complete analytic solution for the diffusion constant of the repton model for all system sizes.

Note added. Recently we became aware of Ref. [15], in which exact values for the diffusion constant of the repton model are calculated for values of $N$ up to 20 using a method entirely different from the one described here. We thank Michael Prähofer for bringing this to our attention.

\section{ACKNOWLEDGMENTS}

The authors would like to thank Ben Widom and Mike Widom for useful comments and illuminating conversations. G.T.B. would like to thank the Santa Fe Institute for their hospitality while this work was carried out. This research was funded in part by the DOE under Grant No. DE-FG029OER40542 and by the Santa Fe Institute and DARPA under Grant No. ONR N00014-95-1-0975.
[1] P. G. de Gennes, J. Chem. Phys. 55, 572 (1971).

[2] M. Rubinstein, Phys. Rev. Lett. 59, 1946 (1987).

[3] T. A. J. Duke, Phys. Rev. Lett. 62, 2877 (1989).

[4] G. T. Barkema and M. E. J. Newman, Physica A (to be published).

[5] G. T. Barkema, C. Caron, and J. F. Marko, Biopolymers 38, 665 (1996).

[6] H. Hervet and C. P. Bean, Biopolymers 26, 727 (1987).

[7] J. M. J. van Leeuwen, J. Phys. I 1, 1675 (1991); J. M. J. van Leeuwen and A. Kooiman, Physica A 184, 79 (1992).

[8] M. Prähofer, Diplomarbeit, Ludwig-Maximilians-Universität München (1994).
[9] B. Widom, J. L. Viovy, and A. D. Defontaines, J. Phys. I 1, 1759 (1991).

[10] I. Szleifer and R. H. Bisseling (unpublished).

[11] G. T. Barkema, J. F. Marko, and B. Widom, Phys. Rev. E 49, 5303 (1994).

[12] B. Derrida, M. R. Evans, V. Hakim, and V. Pasquier, J. Phys. A 26, 1493 (1993).

[13] R. B. Stinchcombe and G. M. Schütz, Phys. Rev. Lett. 75, 140 (1995).

[14] It can be shown that the rank $R_{N}$ of the matrix for a system of $N$ reptons obeys the relation $R_{N}=4\left(R_{N-1}-R_{N-2}\right)$, and given this result the expression for $R_{N}$ above follows by induction.

[15] M. Prähofer and H. Spohn, Physica A 233, 191 (1996). 